\title{
Idiopathic thrombocytopenic purpura with venous thrombosis: A case report
}

\author{
Venöz trombozlu idiopatik trombositopenik purpura: Bir olgu sunumu
}

\author{
Gül İlhan, Selami K. Toprak, Neslihan Andıç, Sema Karakuş \\ Department of Hematology, Başkent University School of Medicine, Ankara, Turkey
}

\section{To the Editor,}

We report a case with idiopathic thrombocytopenic purpura (ITP) who had venous thrombosis. A 59-year-old man with ITP was referred to our hospital because of chest pain. We planned splenectomy after intravenous immunoglobulin (IVIG) treatment because he showed no response to corticosteroid. During IVIG treatment and after splenectomy, venous thrombosis occurred. He was found heterozygous for factor V Leiden, prothrombin (PT) $20210 \mathrm{G}>\mathrm{A}$ and methyltetrahydrofolate reductase (MTHFR) C67TT.

Idiopathic thrombocytopenic purpura (ITP) is an acquired autoimmune disorder characterized by thrombocytopenia and mucocutaneous bleeding and it may occur acutely, chronically or recurrently. While the acute form of the disease is seen in childhood, the chronic form is generally seen in adults. Splenectomy is recommended for patients who do not respond to primary therapy (glucocorticoids, IVIG and/or anti-D) and who have clinical symptoms of bleeding when there is no contraindication for surgery. Thromboembolic complications after splenectomy are described in $10 \%$ of patients with ITP and may be seen as portal vein thrombosis, pulmonary embolism or crural deep vein thrombosis [1]
A 59-year-old man was referred to our hospital because of chest pain. No peripheral lymphadenopathy or petechial or purpuric lesions were determined on his physical examination. Routine laboratory tests showed thrombocytopenia $\left(4000 / \mathrm{mm}^{3}\right)$ confirmed by peripheral blood smear. Etiologic tests were as follows: HBs Ag: (-), antiHBs: (-), antiHCV: (-), antiHIV: (-), antinuclear antibody (ANA): (-), anticardiolipin IgM: 3.1 (0-7) MPL U/ml, and anticardiolipin IgG: $3.2(0-10) \mathrm{GPL} \mathrm{U} / \mathrm{ml}$. Abdominal ultrasonography showed no organomegaly or lymphadenopathy. Bone marrow aspiration smear analysis showed that development of myeloid and erythroid series was normal, both young and mature forms of megakaryocytes were seen, and counts were elevated. Platelet release was decreased. We accepted this case as ITP. Since his platelet count showed no response to oral prednisolone treatment, pulse steroid was administered at a dose of $1 \mathrm{~g} /$ day for 3 days, but again there was no response in platelet count, and splenectomy was planned. In the preoperative period just after IVIG treatment, pain and hyperemia associated with the venous catheter line developed. Upper extremity Doppler ultrasonography showed acute thrombosis in the left brachial and axillary veins. At this time, his 
thrombocyte count was $18,000 / \mathrm{mm}^{3}$. Although IVIG can rarely cause deep vein thrombosis, we studied all thrombophilic tests. Results of these tests were as follows: protein S: 82 (6-140\%), protein C: 122 (70-140\%), antithrombin III (ATIII): 139 (80-120), lupus anticoagulant: 34.2 (20-60), and fasting homocysteine: 10.36 (4.5-15) umol/L. At the one-month follow-up after splenectomy, he complained of swelling and pain on his right leg. Doppler ultrasonography showed acute-subacute thrombosis in the right popliteal and left saphenous veins. At this time, his platelet count was $230,000 / \mathrm{mm} 3$. He was found heterozygous for factor V Leiden, PT 20210 G>A and MTHFR C677T genes. We planned anticoagulation with lifelong warfarin.

In patients presenting with thrombocytopenia, diagnosis of ITP is made by exclusion of conditions that may cause thrombocytopenia, which include: drug use (generally heparin, alcohol, quinine/quinidine, sulphonamides), bacterial infections, viral infections (HIV, hepatitis, cytomegalovirus, EpsteinBarr virus), rickettsia infections, mycoplasma infections, lymphoproliferative diseases (chronic lymphocytic leukemia, large granular lymphocytic leukemia, lymphoma), autoimmune diseases (especially systemic lupus erythematosus, antiphospholipid antibody (APA) syndrome), disseminated intravascular coagulopathy, hemolytic uremic syndrome, thrombotic thrombocytopenic purpura, preeclampsia/eclampsia, HELLP syndrome, gestational thrombocytopenia, and hypersplenism. Patients who develop thrombosis after presenting with thrombocytopenia should be investigated for APA syndrome. This syndrome is characterized by thrombosis in the arterial and venous systems and persistently high levels of antiphospholipid antibodies. APA syndrome is a multisystem disease that may be accompanied by thrombocytopenia and recurrent fetal losses. In our case, both anticardiolipin antibodies and lupus anticoagulant levels were normal at the time of diagnosis. Our patient was taking IVIG when the first thrombosis attack occurred. Especially thromboembolic events may be seen in patients with advanced age, diabetes mellitus, hypertension, and dyslipidemia or in patients given high doses of IVIG rapidly [2]. Thromboembolic events have been reported in $10 \%$ of patients undergoing splenectomy for hematologic diseases. Causes of thrombosis seen in these patients have not been clarified yet. The reported etiologic factors are damage to the portal vein and mesenteric veins during operation, hypercoagulability (protein $\mathrm{C}$, protein S, ATIII deficiency, etc.) and postsplenectomy thrombocytosis. In addition, according to some studies, hematologic disease and spleen size may play a role in thrombosis development. Postsplenectomy portal vein and mesenteric thrombosis is most often seen following splenectomy for myeloproliferative disorders and almost never after trauma [3-5]. Because our patient had thrombosis attacks in the upper and lower extremities, although he was thrombocytopenic when the first attack occurred, we investigated hereditary thrombophilia and found three hereditary thrombophilic risk factors. Thus, anticoagulant therapy was planned despite his advanced age.

\section{Conflict of Interest}

No author of this paper has a conflict of interest, including specific financial interests, relationships, and/or affiliations relevant to the subject matter or materials included in this manuscript.

Oral informed consent was obtained from the patient.

\section{References}

1. Mohren M, Markmann I, Dworschak U, Franke A, Maas C, Mewes S, Weiss $G$, Jentsch-Ullrich $K$. Thromboembolic complications after splenectomy for hematologic diseases. Am J Hematol 2004; 76:143-7.

2. Orbach H, Katz U, Sherer Y, Shoenfeld Y. Intravenous immunoglobulin: adverse effects and safe administration. Clin Rev Allergy Immunol 2005;29:173-84.

3. Solson MM, Ilada PB, Alpergen KN. Portal vein thrombosis. Surg Endosc 2003;17:1322.

4. Silberstein E, Smolikov A, Levi I. Portal and superior mesenteric vein thrombosis after splenectomy for idiopathic thrombocytopenic purpura. Harefuah 1999; 136:364-6, 418.

5. Valeri A, Venneri F, Presenti L, Nardi F, Grossi A, Borrelli D. Portal thrombosis. A rare complication of laparoscopic splenectomy. Surg Endosc 1998;12:1173-6. 